\title{
Oncofertility: combination of ovarian stimulation with subsequent ovarian tissue extraction on the day of oocyte retrieval
}

\author{
Ralf Dittrich ${ }^{1 * \dagger}$, Laura Lotz ${ }^{1 \dagger}$, Andreas Mueller ${ }^{2}$, Inge Hoffmann ${ }^{1}$, David L Wachter ${ }^{3}$, Kerstin U Amann ${ }^{3}$,
} Matthias W Beckmann ${ }^{1}$ and Thomas Hildebrandt ${ }^{1}$

\begin{abstract}
Background: New anticancer treatments have increased survival rates for cancer patients, but often at the cost of sterility. Several strategies are currently available for preserving fertility. However, the chances of achieving a pregnancy with one technique are still limited. A combination of methods is therefore recommended in order to maximize women's chances of future fertility. In this retrospective study, ovarian stimulation with subsequent ovarian tissue extraction on the day of oocyte retrieval were combined and the quality of the ovarian tissue, the numbers and quality of oocytes, time requirements, and the safety of the strategy were examined.

Methods: Fourteen female patients suffering from malignant diseases underwent one in vitro fertilization cycle. Different stimulation protocols were used, depending on the menstrual cycle. Transvaginal oocyte retrieval was scheduled 34-36 h after human chorionic gonadotropin administration. Immediately afterwards, ovarian tissue was extracted laparoscopically.
\end{abstract}

Results: A mean of 10 oocytes were retrieved per patient, and $67 \%$ of the oocytes were successfully fertilized using intracytoplasmic sperm injection. No periprocedural complications and no complications leading to postponement of the start of chemotherapy occurred. The ovarian tissues were of good quality, with a normal age-related follicular distribution and without carcinoma cell invasion.

Conclusions: An approach using ovarian stimulation first, followed by laparoscopic collection of ovarian tissue, is a useful strategy for increasing the efficacy of fertility preservation techniques. The ovarian tissue is not affected by prior ovarian stimulation.

Keywords: Fertility preservation, Ovarian tissue, Ovarian stimulation, IVF, Oocyte cryopreservation, Ovarian tissue cryopreservation

\section{Background}

In recent years, progress in the diagnosis and treatment of oncological diseases has led to considerable improvements in the survival prognosis, particularly in children and adolescent cancer patients. Unfortunately, aggressive chemotherapy and radiotherapy often cause infertility due to massive destruction of the ovarian reserve, resulting in premature ovarian failure. These women

\footnotetext{
* Correspondence: ralf.dittrich@uk-erlangen.de

${ }^{\dagger}$ Equal contributors

'Department of Obstetrics and Gynecology, Erlangen University Hospital, Universitätsstrasse 21-23, Erlangen 91054, Germany

Full list of author information is available at the end of the article
}

have to face years of hormone replacement therapy and the prospect of infertility, which causes psychological stress $[1,2]$.

Several strategies are currently available for preserving fertility, depending on the risks and probability of gonadal failure, the patient's general health at diagnosis, and the partner's status. These strategies include transposition of the ovaries before radiotherapy, ovarian stimulation followed by cryopreservation of fertilized oocytes or unfertilized oocytes, cryopreservation of in vitro-matured oocytes, cryopreservation and transplantation of ovarian tissue, and administration of gonadotropin-releasing hormone $(\mathrm{GnRH})$ agonists $[3,4]$.

\section{Biomed Central}


Although the variety of fertility preservation strategies available enables cancer patients to have children using their own gametes after overcoming their disease, most of these techniques are still experimental and the efficacy of the individual techniques is limited. A combination of methods is therefore recommended in order to maximize women's chances of future fertility [5].

In this study, ovarian stimulation and ovarian tissue cryopreservation were combined as a strategy for fertility preservation in cancer patients. The aim was to evaluate whether ovarian stimulation affects the quality of ovarian tissue. The numbers and quality of oocytes, time requirements, and the safety of this strategy were also examined.

\section{Methods}

Fourteen patients between 24 and 35 years of age (median 29) were included in the retrospective study. They were all suffering from malignant diseases (Table 1) and wanted to preserve their fertility for a future pregnancy. None of the patients had been treated with chemotherapy or radiotherapy before the fertility preservation procedure. All patients provided informed consent for ovarian stimulation and ovarian tissue cryopreservation, after receiving counseling on alternative options for fertility preservation techniques. Three patients also wished to be treated with $\mathrm{GnRH}$ agonists during chemotherapy.

\section{Ovarian stimulation}

All of the patients had regular menstrual cycles before chemoradiotherapy. The phase of the menstrual cycle was evaluated using the onset of the last menstrual period, ultrasonography, and progesterone concentrations. Patients who were stimulated during the follicular phase received either a short "flare-up" protocol or a $\mathrm{GnRH}$-antagonist protocol [6,7]. In the case of a single patient, stimulation was started in the luteal phase with a modified GnRH-antagonist protocol [8]. In one case, ovarian stimulation was carried out with letrozole in combination with a $\mathrm{GnRH}$-antagonist protocol, due to estrogen receptor-positive breast cancer $[9,10]$.

Follicular growth was monitored using vaginal ultrasound and measurement of $17 \beta$-estradiol $\left(\mathrm{E}_{2}\right)$ levels. The gonadotropin dosage was adjusted according to the preantral follicle count and follicle growth. A single dose of recombinant human chorionic gonadotropin (hCG) was administered when the lead follicle had a mean diameter of $15-18 \mathrm{~mm}$.

\section{Oocyte and ovarian tissue collection}

Transvaginal oocyte retrieval was scheduled 34-36 h after hCG administration and was performed with the patient under general anesthesia. Immediately afterwards, ovarian tissue was extracted laparoscopically; the stimulated ovary was divided along the longitudinal midline with scissors, without the use of any diathermy. During this procedure, an effort was made to avoid coming too close to the ovarian mesentery, containing the ovarian vessels. In this way, the anti-mesenteric half of one ovary was separated and retrieved for cryopreservation, while the other half was left in situ. For hemostasis

Table 1 Main characteristics and stimulation outcome in patients who provided informed consent for ovarian stimulation and ovarian tissue cryopreservation

\begin{tabular}{|c|c|c|c|c|c|}
\hline Patient & Cancer type & Age & $\begin{array}{c}\text { Days of } \\
\text { stimulation }\end{array}$ & Aspirated oocytes (n) & $\begin{array}{l}\text { Cryopreserved zygotes } \\
\text { after ICSI (n) }\end{array}$ \\
\hline 1 & Acute myelogenous leukemia & 28 & 9 & 5 & 5 \\
\hline 2 & Hodgkin's lymphoma & 25 & 6 & 7 & 4 \\
\hline 3 & Cervix carcinoma & 31 & 10 & 10 & 5 \\
\hline 4 & Leiomyosarcoma & 26 & 11 & 13 & 11 \\
\hline 5 & Breast carcinoma & 35 & 10 & 7 & 5 \\
\hline 6 & Breast carcinoma & 31 & 8 & 6 & $4^{a}$ \\
\hline 7 & Mesothelioma & 26 & 10 & 10 & 8 \\
\hline 8 & Hodgkin's lymphoma & 24 & 8 & 6 & 4 \\
\hline 9 & Hodgkin's lymphoma & 30 & $9^{a}$ & 25 & $25^{b}$ \\
\hline 10 & Breast carcinoma & 32 & 6 & 7 & $7^{b, c}$ \\
\hline 11 & Breast carcinoma & 30 & 10 & 10 & 5 \\
\hline 12 & Hodgkin's lymphoma & 25 & 13 & 4 & 3 \\
\hline 13 & Non-Hodgkin's lymphoma & 27 & 11 & 14 & $14^{b, c}$ \\
\hline 14 & Breast carcinoma (hormone receptor-positive) & 33 & 11 & 13 & 7 \\
\hline
\end{tabular}

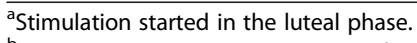

${ }^{\text {b }}$ No ICSI; oocytes were cryopreserved in an unfertilized state.

${ }^{\mathrm{c}}$ Received gonadotropin-releasing hormone agonists during cancer treatment. 
after this, circumscribed bleeding from the ovarian tissue was coagulated using bipolar diathermy. This was necessary at points along the surface of the ovary and on the ovarian septa between the former follicles.

The ovarian cortices were cryopreserved using a slow freezing protocol and an open freezing system [11]. Prior to freezing, a small biopsy of the ovarian tissue was examined histologically to assess follicle density and exclude involvement of the tissue by malignancy.

The mature (metaphase II, MII) oocytes were fertilized by intracytoplasmic sperm injection (ICSI) to avoid the risk of fertilization failure with in vitro fertilization (IVF) and cryopreserved at the pronuclear (PN) stage using a slow freezing protocol in accordance with the patient's request and with German national law. If the patient did not have a partner or the patient requested it, all oocytes were cryopreserved in an unfertilized state using a slow freezing protocol.

\section{Results}

All of the women underwent one IVF cycle. The median period of the hormonal stimulation cycle, between the start of hormonal stimulation and hCG administration, was 10 days (range 6-13 days). The average number of oocytes retrieved per patient was 10 , and $67 \%$ of the oocytes were successfully fertilized using ICSI. In three cases, the oocytes were cryopreserved in an unfertilized state (mean number of unfertilized oocytes 15) (Table 1).

The transvaginal oocyte retrieval and extraction of the ovarian tissue immediately afterward were uneventful in all cases. No perioperative complications such as severe bleeding occurred. The fertility preservation procedures did not lead to postponement of the start of chemotherapy for any of the patients; however, one patient developed a mild ovarian hyperstimulation syndrome (OHSS).

The prefreezing histological sections from the human ovarian grafts had a normal histological appearance. The follicular count showed a normal age-related follicular distribution, with the vast majority of follicles being primordial. No carcinoma cells were seen in any of the hematoxylin-eosin-stained slides examined (Figure 1).

\section{Discussion}

Ovarian stimulation, followed by intracytoplasmic sperm injection and cryopreservation of embryos, is currently the most successful procedure for fertility preservation in newly diagnosed cancer patients. Depending on the patient's age, a survival rate of the embryos following thawing of $35-90 \%$, an implantation rate of up to $30 \%$, and a cumulative pregnancy rate of $30-40 \%$ can be achieved [12,13].

Freezing unfertilized oocytes is also a promising option for preserving fertility today. Oocyte banking does not require any partner or sperm donor and it may also

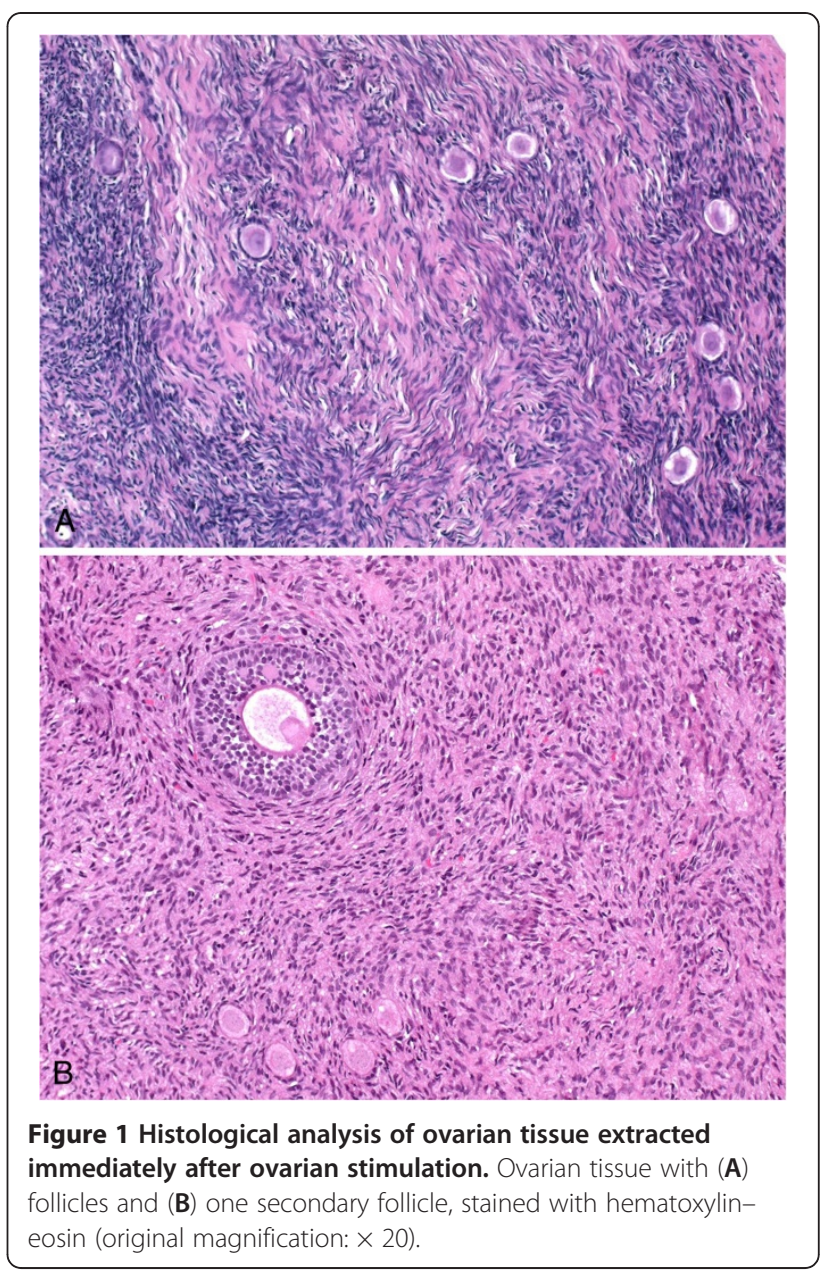

accord better with various religious or ethical considerations than embryo freezing. With recent improvements in freeze-thaw protocols such as vitrification, promising results with more than $60 \%$ of mature oocytes surviving after thawing and subsequent fertilization have been reported - rates comparable with fresh oocytes [14,15].

For either of these methods to be successful, however, appropriate quantities of oocytes have to be obtained. In addition, because the time frame up to the initiation of chemotherapy and/or radiotherapy is limited, usually only one IVF cycle can be carried out, and the numbers of oocytes or embryos cryopreserved are consequently often not sufficient for several transfer attempts. For maximum effectiveness, combinations with other fertility preservation techniques therefore need to be considered.

Cryopreservation of ovarian tissue offers an effective combination. Cryopreservation of ovarian tissue before oncologic treatment has recently become one of the most promising techniques for preserving fertility. It allows storage of a large number of primordial and primary follicles. It can be carried out rapidly at any time in the menstrual cycle without delaying the oncological treatment 
and provides a unique option for preserving fertility in prepubertal or premenarchal female patients [16]. However, the method is surgically invasive and there is a potential risk that malignant cells in the frozen tissue may lead to recurrence of the primary disease after transplantation. For most conditions, however, the risk is low and is presumably related to the stage of disease at the time of ovarian tissue cryopreservation, although considerable caution is advisable with cryoconserved tissue from patients with leukemia, borderline ovarian tumor, or with a high risk of ovarian metastases (e.g., in adenocarcinoma of the cervix or stage III-IV breast cancer) [17]. A total of 20 live births have been reported to date after orthotopic transplantation of cryopreserved ovarian tissue [18-21]. Although cryopreservation of ovarian tissue is still considered experimental, the technique is now gaining worldwide acceptance.

In the cancer patients included in the present study, ovarian stimulation was carried out first, followed by laparoscopic collection of the ovarian tissue. Although it has been reported that ovarian tissue is of poor quality after ovarian stimulation [22], no data on this topic have so far been published. Histological examination of the ovarian tissue showed a normal age-related follicle distribution. No histological differences were found from ovarian tissue from patients who underwent ovarian tissue cryopreservation in our department without prior ovarian stimulation. Nor was any correlation noted between the numbers of oocytes retrieved and the follicle distribution in the ovarian tissue. In patients with fewer retrieved oocytes, the numbers of follicles were similar to those in patients with a high response to ovarian stimulation.

The ovarian response to stimulation is crucial for successful fertility preservation, and there has been concern regarding the ovarian response to ovarian stimulation in cancer patients. In the present study, different stimulation protocols were used due to the different starting days for stimulation. Adequate numbers of oocytes were retrieved within 2 weeks. The average number of oocytes retrieved per patient was 10 , and $67 \%$ of the oocytes were successfully fertilized. This is in accordance with recent studies that have reported no significant changes in the ovarian reserve or response to gonadotropins in patients with various types of cancer $[19,20]$. However, other studies have reported a poorer ovarian response in cancer patients undergoing IVF treatment protocols $[17,18]$. The published data on this topic are still inconsistent.

The present group of patients included five women with breast cancer, one of whom had estrogen receptor-positive breast cancer. Concerns have been raised regarding the use of controlled ovarian stimulation in patients with hormone-dependent tumors, due to inadequate data on short-term increases in hormonal effects on the tumor. Moreover, as animal models suggest, estrogen may also play a role in stimulating the growth of estrogen receptor-negative breast cancers [23]. Conventional stimulation protocols with gonadotropins are therefore modified to include administration of the aromatase inhibitor letrozole $[9,24]$ or the selective estrogen modulator tamoxifen [25]. These protocols have been used with success in reducing the estradiol excesses that are normally seen with conventional protocols, and short-term follow-up data for these protocols have not shown any detrimental effects on survival [10].

The risk of ovarian hyperstimulation syndrome (OHSS) is a known complication of controlled ovarian stimulation. One patient in the present study developed a mild OHSS, but the start of cancer treatment did not have to be postponed in any of the patients. The overall risk of severe OHSS is low, and in cancer patients it is also reduced, given that pregnancy will not occur; however, the risk should not be underestimated. Careful selection of the gonadotropin starting dosage, close monitoring, and step-down dosing are critical for avoiding complications. Triggering using a GnRH agonist alone or together with low-dose hCG might potentially further reduce the risk of hyperstimulation [26,27].

A potential side effect of the subsequent use of oocyte retrieval and ovarian tissue extraction may be bleeding in the residual ovarian tissue. Stimulated ovaries are more fragile than unstimulated ovarian tissue, which has a more compact structure. Stimulated ovaries have to be handled with greater care in comparison with unstimulated ovaries, to avoid injuries to the surface and to minimize possible tissue damage and bleeding. However, no side effects of this type were observed in any of the patients.

Several attempts have been made to improve the effectiveness of fertility preservation programs by combining different techniques. Removing ovarian tissue first and starting ovarian stimulation approximately $1-2$ days later is an effective alternative approach. The partial removal of ovarian tissue does not substantially affect the average number or quality of oocytes retrieved after ovarian stimulation [22].

The combination of cryopreservation of ovarian tissue before chemotherapy and ovarian stimulation after the start of chemotherapy should no longer be carried out, as the efficacy of IVF is dramatically reduced even after one round of chemotherapy and high rates of malformation of offspring after treatment with alkylating agents have been demonstrated experimentally $[28,29]$.

If there is no time for ovarian stimulation, cryopreservation of oocytes retrieved during dissection of resected ovarian tissue has also been reported as a potential strategy for preserving fertility in patients with cancer. The 
combination of in vitro maturation (IVM) with oocyte cryopreservation prevents any delay in cancer treatment and avoids the risks associated with high estradiol levels in hormone-sensitive tumors [30]. Although healthy infants have been born following IVM, implantation and pregnancy rates are generally lower than for IVF with mature oocytes [31,32].

Administration of GnRH-agonist analogs, in an attempt to reduce the gonadotoxic effects of chemotherapy by simulating a prepubertal hormonal milieu, is another fertility preservation method and should be combined with other fertility-protecting measures as well if possible. Although conclusive proof is still awaited, there is increasing evidence that GnRH agonists are effective in protecting the ovaries [33]. Administration of these agents may be considered on an individual basis, as the method is safe, noninvasive, and easy to administer.

\section{Conclusions}

Fertility-preserving procedures should be offered to all patients facing fertility loss before cytotoxic treatment is administered. The decision as to which fertility preservation treatment is most suitable in the patient's individual situation has to be made during a personal discussion with her and requires intensive interdisciplinary discussion, including oncologists, radiotherapists, and reproductive medicine specialists. A combination of fertility preservation techniques increases the efficacy of the procedure and gives young cancer patients the best chance for future fertility $[5,34]$.

\section{Abbreviations}

GnRH: Gonadotropin-releasing hormone; hCG: human chorionic gonadotropin; ICSI: Intracytoplasmic sperm injection; IVF: in vitro fertilization; IVM: in vitro maturation; OHSS: Ovarian hyperstimulation syndrome; PN: Pronuclear (stage).

\section{Competing interests}

The authors hereby declare that they have no competing interests.

\begin{abstract}
Authors' contributions
$L L, A M, R D$, and $T H$ designed the study and analyzed the data. $L L$ and $R D$ wrote the manuscript. TH collected the data. AM, TH, and MWB carried out the operations. DW, LL, KUA, and $I H$ carried out the histological work and analysis. MWB and RD supervised the study. All of the authors read and approved the final manuscript.
\end{abstract}

\section{Acknowledgments}

Research for this study was supported by grants from the Wilhelm Sander Foundation (reference no. 2008.086.1), Munich, Germany, and the Deutsche Forschungsgemeinschaft (DI 1525/4-1).

\section{Author details}

'Department of Obstetrics and Gynecology, Erlangen University Hospital, Universitätsstrasse 21-23, Erlangen 91054, Germany. ${ }^{2}$ Department of Obstetrics and Gynecology, Karlsruhe Hospital, Moltkestrasse 90, Karlsruhe 76133, Germany. 'Department of Pathology, Erlangen University Hospital, Krankenhausstrasse 8-10, Erlangen 91054, Germany.

Received: 5 November 2012 Accepted: 19 February 2013 Published: 5 March 2013

\section{References}

1. Dittrich R, Maltaris T, Hoffmann I, Oppelt PG, Beckmann MW, Mueller A: Fertility preservation in cancer patients. Minerva Ginecol 2010, 62:63-80.

2. Lawrenz B, Jauckus J, Kupka M, Strowitzki T, Wolff M: Fertility preservation in $>1,000$ patients: patient's characteristics, spectrum, efficacy and risks of applied preservation techniques. Arch Gynecol Obstet 2011, 283:651-656.

3. Rodriguez-Wallberg KA, Oktay K: Options on fertility preservation in female cancer patients. Cancer Treat Rev 2012, 38:354-361.

4. Maltaris T, Beckmann MW, Dittrich R: Fertility preservation for young female cancer patients. In Vivo 2009, 23:123-130.

5. Huser M, Zakova J, Smardova L, Crha I, Janku P, Hudecek R, Ventruba P: Combination of fertility preservation strategies in young women with recently diagnosed cancer. Eur J Gynaecol Oncol 2012, 33:42-50.

6. Del Gadillo JCB, Siebzehnrübl E, Dittrich R, Wildt L, Lang N: Comparison of GnRH agonists and antagonists in unselected IVF/ICSI patients treated with different controlled ovarian hyperstimulation protocols: a matched study. Eur J Obstet Gynecol Reprod Biol 2002, 102:179-183.

7. Bodri D, Vernaeve V, Guillén JJ, Vidal R, Figueras F, Coll O: Comparison between a $\mathrm{GnRH}$ antagonist and a $\mathrm{GnRH}$ agonist flare-up protocol in oocyte donors: a randomized clinical trial. Hum Reprod 2006, 21:2246-2251.

8. von Wolff M, Thaler CJ, Frambach T, Zeeb C, Lawrenz B, Popovici RM, Strowitzki T: Ovarian stimulation to cryopreserve fertilized oocytes in cancer patients can be started in the luteal phase. Fertil Steril 2009, 92:1360-1365.

9. Oktay K, Hourvitz A, Sahin G, Oktem O, Safro B, Cil A, Bang H: Letrozole reduces estrogen and gonadotropin exposure in women with breast cancer undergoing ovarian stimulation before chemotherapy. J Clin Endocrinol Metab 2006, 91:3885-3890.

10. Azim AA, Costantini-Ferrando M, Oktay K: Safety of fertility preservation by ovarian stimulation with letrozole and gonadotropins in patients with breast cancer: a prospective controlled study. J Clin Oncol 2008, 26:2630-2635

11. Dittrich R, Maltaris T: A simple freezing protocol for the use of an open freezing system for cryopreservation of ovarian tissue. Cryobiology 2006, $52: 166$.

12. Ata B, Chian RC, Tan SL: Cryopreservation of oocytes and embryos for fertility preservation for female cancer patients. Best Pract Res Clin Obstet Gynaecol 2010, 24:101-112.

13. Sonmezer M, Oktay K: Fertility preservation in female patients. Hum Reprod Update 2004, 10:251-266.

14. Noyes N, Knopman JM, Melzer K, Fino ME, Friedman B, Westphal LM: Oocyte cryopreservation as a fertility preservation measure for cancer patients. Reprod Biomed Online 2011, 23:323-333.

15. Yoon TK, Lee DR, Cha SK, Chung HM, Lee WS, Cha KY: Survival rate of human oocytes and pregnancy outcome after vitrification using slush nitrogen in assisted reproductive technologies. Fertil Steril 2007, 88:952-956

16. von Wolff M, Donnez J, Hovatta O, Keros V, Maltaris T, Montag M, Salle B, Sonmezer M, Andersen CY: Cryopreservation and autotransplantation of human ovarian tissue prior to cytotoxic therapy-a technique in its infancy but already successful in fertility preservation. Eur J Cancer 2009, 45:1547-1553.

17. Rosendahl M, Greve T, Andersen CY: The safety of transplanting cryopreserved ovarian tissue in cancer patients: a review of the literature. J Assist Reprod Genet 2013, 30:11-24.

18. Donnez J, Jadoul P, Pirard C, Hutchings G, Demylle D, Squifflet J, Smitz J, Dolmans MM: Live birth after transplantation of frozen-thawed ovarian tissue after bilateral oophorectomy for benign disease. Fertil Steril 2012, 98:720-725.

19. Silber S, Kagawa N, Kuwayama M, Gosden R: Duration of fertility after fresh and frozen ovary transplantation. Fertil Steril 2010, 94:2191-2196.

20. Revel A, Laufer N, Ben Meir A, Lebovich M, Mitrani E: Micro-organ ovarian transplantation enables pregnancy: a case report. Hum Reprod 2011, 26:1097-1103.

21. Dittrich R, Lotz L, Keck G, Hoffmann I, Mueller A, Beckmann MW, van der Ven $\mathrm{H}$, Montag M: Live birth after ovarian tissue autotransplantation following overnight transportation before cryopreservation. Fertil Steril 2012, 97:387-390

22. Huober-Zeeb C, Lawrenz B, Popovici RM, Strowitzki T, Germeyer A, Stute P, von Wolff M: Improving fertility preservation in cancer: ovarian tissue 
cryobanking followed by ovarian stimulation can be efficiently combined. Fertil Steril 2011, 95:342-344.

23. Gupta PB, Proia D, Cingoz O, Weremowicz J, Naber SP, Weinberg RA, Kuperwasser C: Systemic stromal effects of estrogen promote the growth of estrogen receptor-negative cancers. Cancer Res 2007, 67:2062-2071.

24. Checa Vizcaíno MA, Corchado AR, Sastre I, Cuadri ME, Comadran MG, Brassesco M, Carreras R: The effects of letrozole on ovarian stimulation for fertility preservation in cancer-affected women. Reprod Biomed Online 2012, 24:606-610.

25. Oktay K, Buyuk E, Davis O, Yermakova I, Veeck L, Rosenwaks Z: Fertility preservation in breast cancer patients: IVF and embryo cryopreservation after ovarian stimulation with tamoxifen. Hum Reprod 2003, 18:90-95.

26. Oktay K, Türkçüoğlu I, Rodriguez-Wallberg KA: GnRH agonist trigger for women with breast cancer undergoing fertility preservation by aromatase inhibitor/FSH stimulation. Reprod Biomed Online 2010 20:783-788

27. Humaidan P: Agonist trigger: what is the best approach? Agonist trigger and low dose hCG. Fertil Steril 2012, 97:529-530.

28. Dolmans MM, Demylle D, Martinez-Madrid B, Donnez J: Efficacy of in vitro fertilization after chemotherapy. Fertil Steril 2005, 83:897-901.

29. Meirow D, Epstein M, Lewis H, Nugent D, Gosden RG: Administration of cyclophosphamide at different stages of follicular maturation in mice: effects on reproductive performance and fetal malformations. Hum Reprod 2001, 16:632-637.

30. Huang JYJ, Tulandi T, Holzer H, Tan SL, Chian RC: Combining ovarian tissue cryobanking with retrieval of immature oocytes followed by in vitro maturation and vitrification: an additional strategy of fertility preservation. Fertil Steril 2008, 89:567-572

31. Fasano G, Moffa F, Dechène J, Englert Y, Demeestere I: Vitrification of in vitro matured oocytes collected from antral follicles at the time of ovarian tissue cryopreservation. Reprod Biol Endocrinol 2011, 9:150

32. Suikkari AM: In-vitro maturation: its role in fertility treatment. Curr Opin Obstet Gynecol 2008, 20:242-248.

33. Badawy A, Elnashar A, El-Ashry M, Shahat M: Gonadotropin-releasing hormone agonists for prevention of chemotherapy-induced ovarian damage: prospective randomized study. Fertil Steril 2009, 91:694-697.

34. von Wolff M, Montag M, Dittrich R, Denschlag D, Nawroth F, Lawrenz B: Fertility preservation in women-a practical guide to preservation techniques and therapeutic strategies in breast cancer, Hodgkin's lymphoma and borderline ovarian tumours by the fertility preservation network FertiPROTEKT. Arch Gynecol Obstet 2011, 284:427-435.

doi:10.1186/1477-7827-11-19

Cite this article as: Dittrich et al:: Oncofertility: combination of ovarian stimulation with subsequent ovarian tissue extraction on the day of oocyte retrieval. Reproductive Biology and Endocrinology 2013 11:19.

\section{Submit your next manuscript to BioMed Central and take full advantage of:}

- Convenient online submission

- Thorough peer review

- No space constraints or color figure charges

- Immediate publication on acceptance

- Inclusion in PubMed, CAS, Scopus and Google Scholar

- Research which is freely available for redistribution 